\title{
Experimental Demonstrations of Electronic Dispersion Compensation for Long-Haul Transmission Using Direct-Detection Optical OFDM
}

\author{
Brendon J. C. Schmidt, Arthur James Lowery, Senior Member, IEEE, and Jean Armstrong, Senior Member, IEEE
}

\begin{abstract}
We present experimental demonstrations using direct-detection and optical-orthogonal frequency division multiplexing (DD-OOFDM) for the compensation of chromatic dispersion in long-haul optical fiber links. Three transmitter designs of varying electrical and optical complexity are used for optical single sideband (OSSB) transmission and the theory behind each design is discussed. The data rates achieved for the three systems are 10, 12, and $20 \mathrm{Gbit} / \mathrm{s}$ for fiber distances between 320 and $400 \mathrm{~km}$. A discussion of system overheads is provided together with simulations of the required optical signal-to-noise ratio (OSNR).
\end{abstract}

Index Terms-Chromatic dispersion, compensation, optical single sideband (OSSB), orthogonal frequency division multiplexing (OFDM).

\section{INTRODUCTION}

O RTHOGONAL frequency division multiplexing (OFDM) has largely replaced serial modulation formats for new broadband wireless communication systems, because it is a simple solution to signal dispersion and because it scales well when data rate increases result in intersymbol interference that affects multiple symbol periods. Recently there has been increasing interest in OFDM for optical fiber applications because as data rates increase, the computational requirements involved in electronic dispersion compensation (EDC) for serial modulation formats become impractical [1]. Recent research has shown that OFDM can be used for the electronic compensation of chromatic dispersion and polarization mode dispersion in single-mode optical fiber systems [2]-[5] and for mode dispersion in multimode systems [6].

One major advantage of the use of EDC rather than dispersion-compensation fiber (DCF) to compensate for chromatic dispersion in long haul optical links is that the amount of compensation can be changed rapidly, simply by adjusting the parameters of the compensation algorithm; no changes are required to outside plant. The first installed long-haul EDC sys-

Manuscript received July 30, 2007; revised October 30, 2007. This work was supported by the Australian Research Council's Discovery funding scheme (DP 0772937).

The authors are with the Department of Electrical and Computer Systems Engineering, Monash University, Melbourne, VIC 3800, Australia (e-mail: Brendon.Schmidt@eng.monash.edu.au; Arthur.Lowery@eng.monash.edu.au; Jean.Armstrong@eng.monash.edu.au).

Color versions of one or more of the figures in this paper are available online at http://ieeexplore.ieee.org.

Digital Object Identifier 10.1109/JLT.2007.913017 tems used electronic predistortion (EPD) [7], [8]. EPD operates at the transmitter, and adjusts the transmitted optical waveform so that the optical waveform at the receiver is optimized for direct-detection. EPD requires a feedback path from receiver to transmitter to optimize the transmitted waveform; so it cannot cope with rapid channel variations and the signal cannot be recovered at points between the transmitter and receiver. EDC systems that use equalization in the receiver overcome these disadvantages.

EDC using OFDM uses receiver based equalization and is scalable. A number of forms of optical OFDM have been proposed recently. Coherent optical OFDM (CO-OFDM) is simply conventional OFDM as used in radio systems with the radio frequency carrier replaced by an optical carrier and a number of patents and patent applications have recognized that conventional OFDM can be applied to optical fiber communications [9]-[13]. CO-OFDM requires coherent reception and because of the sensitivity of OFDM to frequency offset [14] and phase noise, very narrow linewidth lasers are required at the transmitter and receiver and sophisticated tracking algorithms are required to track laser frequency and phase [15]. To overcome these disadvantages a number of new forms of OFDM tailored to optical applications have been described [16]-[19]. Directdetection optical OFDM (DD-OOFDM) improves on previous systems [20], [21] and has been demonstrated experimentally and by simulation to provide a simple and effective solution to chromatic dispersion for long-haul single-mode fiber applications [2], [22], [23].

This paper reports experimental demonstrations of three versions of DD-OOFDM. These have various degrees of optical complexity at the transmitter, but all use a single photodiode direct-detection photoreceiver. As well as compensating for chromatic dispersion, OFDM can correct for linear distortions in the electrical components, including the optical modulator and photodiode. This means DD-OOFDM systems are tolerant of linear imperfections and the experimental systems were implemented using commercial-off-the-shelf components.

This paper is organized as follows. Section II describes the OFDM systems in detail. Section III presents the experimental setup and results. Section IV discusses the stability of the system. Section V discusses the overheads required to compensate for chromatic dispersion. Section VI provides simulated results for the optical signal-to-noise ratio (OSNR) for different system configurations. Section VII summarizes the paper. 


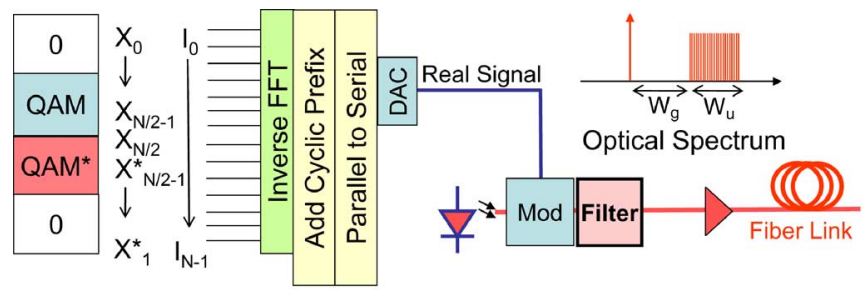

(a)

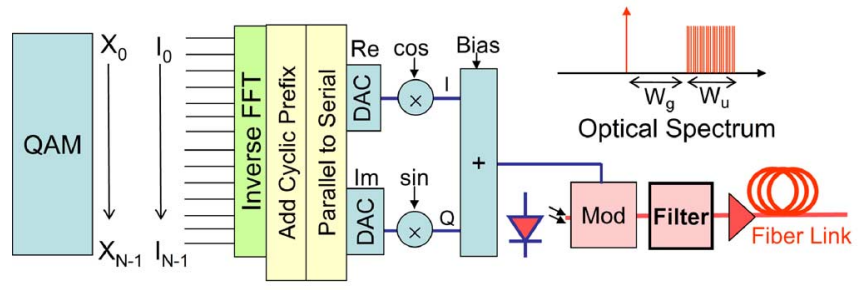

(b)

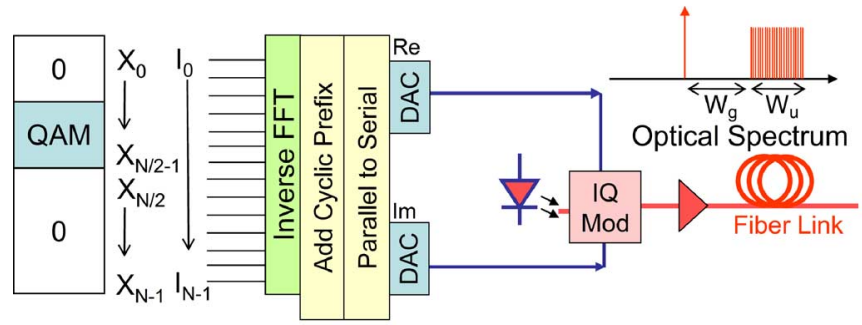

(c)

Fig. 1. DD-OOFDM transmitters: (a) using Hermitian symmetry and an optical filter; (b) using upconversion and an optical filter; (c) using a frequency domain Hilbert transform.

\section{Direct Detection OFDM Systems}

The key advantage of DD-OOFDM is that it can be received using a simple direct detection receiver: no laser is required at the receiver. To achieve this, an optical single sideband (OSSB) OFDM signal and a component at the optical carrier frequency are transmitted. A frequency guard band separates the OFDM signal from the optical carrier. The signal is received by detecting the carrier $\times$ signal mixing products [22]. Chromatic dispersion causes a frequency dependent phase shift of the optical signal. For single sideband optical OFDM systems, with linear field modulation, each OFDM subcarrier is represented by a single optical frequency, so phase shifts in the optical domain translate to phase shifts in the demodulated electrical OFDM subcarriers. These phase shifts can be corrected simply and without SNR penalty in the digital section of the receiver by applying a single complex multiplication for each subcarrier.

\section{A. DD-OOFDM Transmitters}

Fig. 1 shows three different designs for DD-OOFDM transmitters. The digital sections of the transmitter are conventional OFDM systems: an IFFT is used to simultaneously modulate and multiplex the OFDM subcarriers, a cyclic prefix is added and then the signal is converted from parallel to serial format. The digital sections of the transmitters differ only in the way the input vector $\mathbf{X}$ is mapped onto the IFFT input vector $\mathbf{I}$.

Fig. 1(a) shows the details of the first transmitter design. A single input optical modulator is used to generate a double sideband optical signal and then one sideband is suppressed using an optical filter. The electrical input to the optical modulator is a real, baseband signal and only one digital-to-analog converter (DAC) is required. In general, the output of an IFFT is complex rather than real. In systems where a single real output is required, the input vector to the IFFT, $\mathbf{I}$, is constrained to have Hermitian symmetry [6] so that the imaginary component of the IFFT output is zero. Fig. 1(a) shows the input vector $\mathbf{X}=X_{0} \cdots X_{N / 2-1}$ being mapped to the IFFT inputs $\mathbf{I}=$ $I_{0} \cdots I_{N-1}$

$$
I_{0} \cdots I_{N-1}=X_{0} \cdots X_{N / 2-1}, X_{N / 2,} X_{N / 2-1}^{*} \cdots X_{1}^{*}
$$

where $X_{k}^{*}$ denotes the complex conjugate of $X_{k}$ and $N$ is the size of the IFFT. The inputs $X_{0}$ and $X_{N / 2-1}$ which correspond to the dc and Nyquist frequencies are set to zero, as are the inputs corresponding to guard band frequencies. The number of independent complex values transmitted per OFDM symbol depends on the width of the guard band, $W_{G}$. For the case where the guard band is equal to $W_{U}$ the bandwidth used for the OFDM signal, then only $N / 4$ independent complex values can be transmitted per OFDM symbol. The transmitter is simple to set up but if a wavelength-agile (colorless) transmitter is required, a tunable optical filter must be used to track the laser.

The details of the second design are shown in Fig. 1(b). The single DAC of the first design is replaced by two DACs and an electrical RF upconversion stage. This allows the complex baseband OFDM signal to be mixed with an RF carrier before driving the single input optical modulator. The width of the guard band is determined by the RF frequency (not by nulling OFDM inputs) and so all subcarriers except the dc subcarrier can be used to carry data. The analog upconversion allows flexible placement of the signal spectrum relative to the optical carrier and the RF frequency is independent of the DAC sample rate. As in the first design an optical filter is used to suppress one sideband.

For a given data rate, this design requires a DAC sample rate of approximately one quarter that of the first design. But the addition of analog mixers with such high frequency and bandwidth requirements may cause problems with frequency synchronization and inphase (I) and quadrature (Q) balance.

The third transmitter design, which is shown in Fig. 1(c), generates an optical single sideband by using a signal and its Hilbert transform to drive an optical I/Q modulator. A Hilbert transform can be generated simply in an OFDM transmitter, by setting half of the IFFT inputs to zero. In this case the input vector to the IFFT is given by

$$
I_{0} \cdots I_{N-1}=X_{0} \cdots X_{N / 2-1}, 0 \cdots 0 .
$$

The IFFT output is then a single sided, analytic signal and its real and imaginary components are used for the I and Q inputs of the complex optical modulator for OSSB transmission without an optical filter. The frequency guard band is created by setting the corresponding inputs to zero. This design requires two DACs, each with the same sample rate as the first design. 


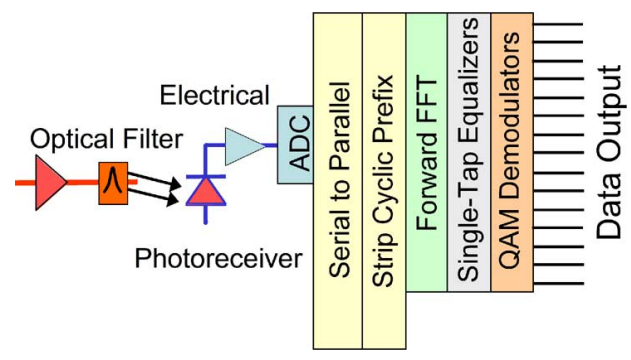

Fig. 2. DD-OOFDM direct detection receiver.

\section{B. Biasing and Linear Field Modulation}

The biasing of the optical modulator plays an important part in system performance. To provide the optimum noise performance, the transmitter optical modulator should be biased for equal carrier and sideband powers [22] as this provides the peak electrical SNR and lowest bit error rate (BER) for a given optical SNR (OSNR). This optimal carrier to sideband power ratio can be achieved by biasing the modulators just above their intensity nulls. Using this bias point also means that the modulator is operating in the linear field region where the optical field is proportional to the input voltage. In the optical OFDM system, this means that the discrete subcarrier frequencies in the baseband electrical domain map to discrete frequencies in the optical domain which after direct detection map back to single electrical subcarrier frequencies. Most conventional optical systems bias the modulator at the quadrature point. But if this bias point is used in an optical OFDM system, the discrete input electrical frequencies map to multiple optical frequencies and direct detection results in high levels of intermodulation distortion (IMD). For OFDM signals which have a high peak to average power ratio (PAPR), the average input drive levels were carefully adjusted to maintain linear field modulation and a reasonable modulator output level.

\section{DD-OOFDM Receiver}

Fig. 2 shows the simple direct detection receiver required for DD-OOFDM. As a result of the square law characteristic of the photodiode, the received signal consists of a number of mixing products. These can be classified into the useful components from which the data is recovered, the unwanted components which fall within band and limit the BER performance of the system and unwanted components which fall out of band. The useful components are the difference terms which result from the mixing of the OFDM sideband and the optical carrier. The unwanted inband terms are the result of carrier $\times$ noise and signal $\times$ noise mixing. If a narrow optical filter is used, only noise from one optical sideband will be detected, however if a wider optical filter is used, noise from both sidebands will mix in with a resulting 3-dB loss in SNR. The use of a frequency guard band means that all of the results of mixing between OFDM subcarriers fall out of band and do not degrade performance. There may also be other second-order effects: for example, nonlinearities in the system or I/Q imbalance in the transmitter will result in other components in the transmitted optical signal which may create other mixing products which degrade performance. The digital section of the receiver is a conventional OFDM system in which the phase and amplitude of each received subcarrier is corrected by the single tap equalizer.

\section{EXPERIMENTAL SETUP AND RESULTS}

A series of experiments were performed to compare the three transmitter designs. In all of the experiments, the OFDM baseband digital signal processing was performed offline using MATLAB. The signals to be transmitted were downloaded into a Tektronix AWG7102 arbitrary waveform generator (AWG) which generated the required analog baseband signals. The received signals were captured using an Agilent 81004A digital sampling oscilloscope (DSO). The OSNR was set by adding a variable ASE noise source to the signal before the final optical amplifier. All OSNR measurements include the entire experimental setup. Standard off-the-shelf, commercial grade, electrical and optical components were used. The main limitation to the achievable data rates for the two digital systems was the sampling rate of the AWG which can generate either two independent $10-\mathrm{GS} / \mathrm{s}$ outputs each with a $5-\mathrm{GHz}$ bandwidth or a single $20-\mathrm{GS} / \mathrm{s}$ output with a $5.8-\mathrm{GHz}$ bandwidth. Chromatic dispersion was not a limitation, because as long as the dispersion is less than the length of the cyclic prefix, chromatic dispersion simply causes a rotation of each OFDM subcarrier which can be corrected by the equalizer. For each system the same QAM constellation was used on each data carrying OFDM subcarrier. The size of constellation for each system was chosen to give a bit error rate averaged over the subcarriers of approximately $1 \times 10^{-3}$.

\section{A. 12-Gbit/s Digital System}

Fig. 3 shows the experimental configuration using the Hilbert transform based colorless transmitter (third transmitter design). The AWG is used in dual output mode to generate an analog signal and its Hilbert transform and these are amplified and used to drive the two inputs of the Sumitomo single sideband modulator (a cascaded triple Mach-Zehnder Interferometer modulator). The Hilbert transform enables a single sideband optical signal to be generated without the need of an optical filter. The optical source for the modulator is a Photonetics tunable laser and the modulated output is buffered and transmitted through five spans of $80 \mathrm{~km}$ of Corning SMF-28e fiber. After each span, the signal is amplified for a target power of $0 \mathrm{dBm}$. After the final span, a Fiber Bragg Grating (FBG) is used as an ASE filter with a 50-GHz bandwidth and the signal is detected by a Discovery Semiconductors DSCR404 photoreceiver. The DSO captures the signal which is later processed using MATLAB.

A 512-point IFFT with no oversampling is used in the transmitter. With a guard band equal in width to the OFDM bandwidth and with the nulling of subcarriers to create the Hilbert transform, this design theoretically allows 128 independent data carrying subcarriers. However in this experiment, a further five subcarriers are not used due to their proximity to a side mode of the external cavity laser. Of the three systems, this one is the least affected by component imperfections and can support the highest order QAM constellation. This is due to the simplicity of the analog electrical and optical components and because the AWG is used within specification. The available 123 subcarriers are modulated with $32 \mathrm{QAM}$, giving a data rate of $12 \mathrm{Gbit} / \mathrm{s}$. A cyclic prefix of 32 samples is used in the two digital systems and a cyclic prefix of 64 samples is used in the system using an $\mathrm{RF}$ carrier. 


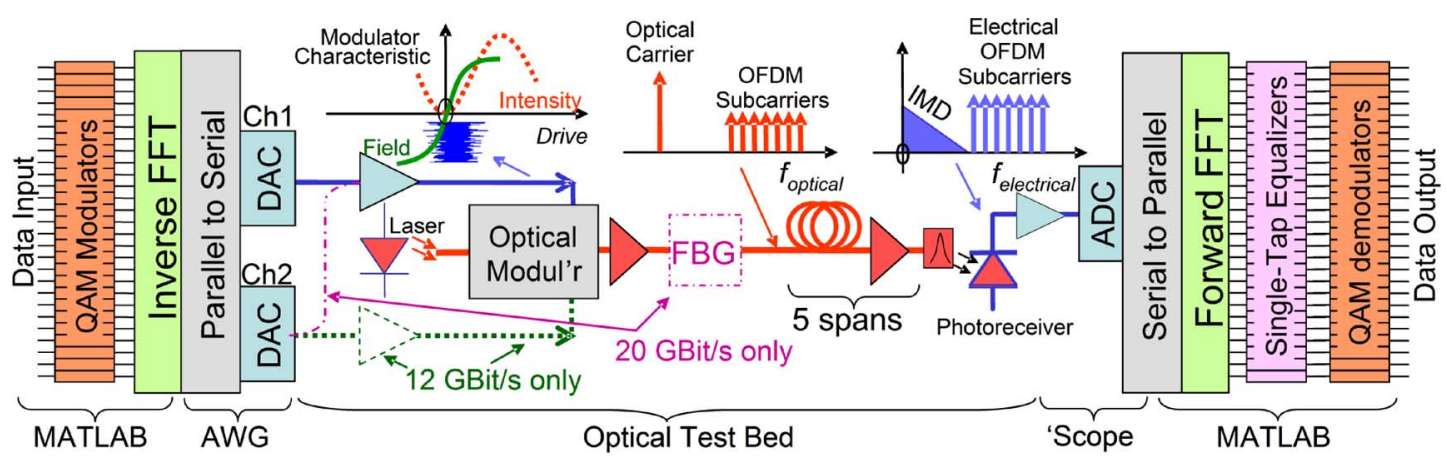

Fig. 3. Schematic of the two digital baseband electrical systems: 12 and $20 \mathrm{Gbit} / \mathrm{s}$.
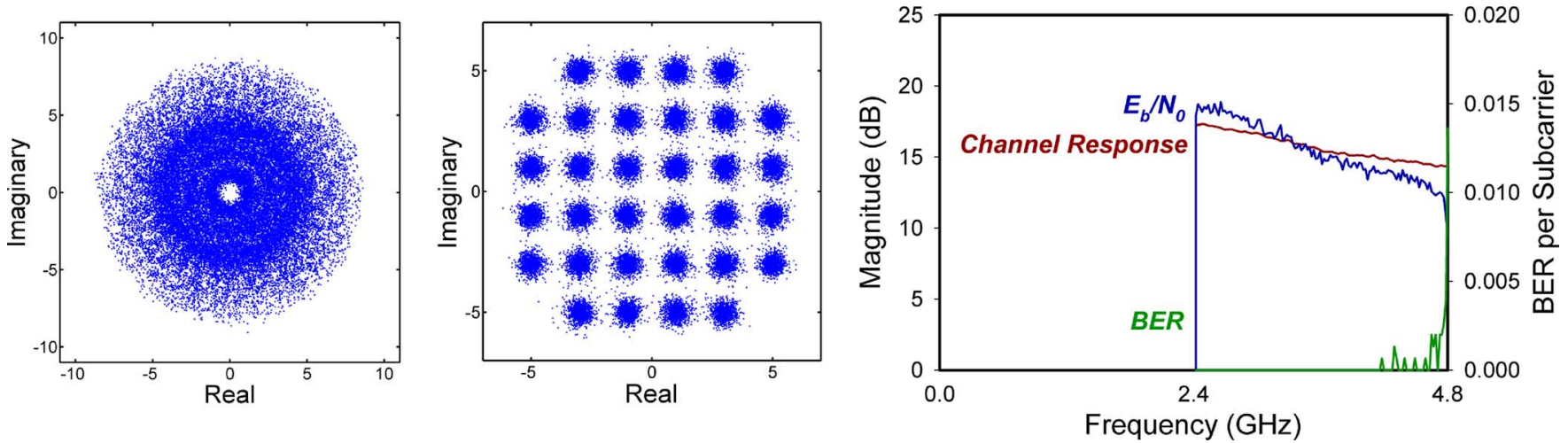

Fig. 4. Results for 12-Gbit/s colorless system using digital Hilbert transform to generate single sideband signal. Unequalized constellation. Equalized constellation. Channel response $\mathrm{E}_{\mathrm{b}} / \mathrm{N}_{0}$ and BER.

Three hundred OFDM symbols (184 500 bits) were transmitted over a distance of $400 \mathrm{~km}$ and recovered with a BER of $3.03 \times 10^{-4}$ at a measured OSNR of $25 \mathrm{~dB}$. The un-equalized and equalized constellations can be seen in Fig. 4. The single tap equalizer function in the OFDM receiver corrects for the amplitude distortions caused by frequency roll-off of the components and the phase distortions caused by chromatic dispersion and OFDM frame timing offsets. The high QAM system also illustrates the low levels of IMD that can be achieved using a modulator biased in the linear optical field region. The required OSNR for the 12-Gbit/s system was $22 \mathrm{~dB}$ for a BER of $1 \times 10^{-3}$. Fig. 4 also shows the relative channel response of the entire system, the received SNR in terms of $E_{\mathrm{b}} / N_{0}$, and the BER as a function of frequency. The figure illustrates the falling response at high frequencies which causes a reduction in the SNR and an increase in the BER on the higher frequency subcarriers.

\section{B. 20-Gbit/s Digital System}

Fig. 3 also shows the experimental configuration for the $20 \mathrm{Gbit} / \mathrm{s}$ digital system which uses a single DAC and an optical filter (first transmitter design). Because only one output is required, the two channels of the AWG are interleaved to increase the sampling rate to $20 \mathrm{GS} / \mathrm{s}$. The I/Q optical modulator of the first experiment is replaced by a Fujitsu MZI modulator. One optical sideband is suppressed using a FBG in transmission mode as an optical filter. Because of the loss in the FBG, the length of the first fiber span is reduced to $30 \mathrm{~km}$. The receiver is the same as for the 12-Gbit/s system, however a tunable optical filter (200-GHz FWHM) is used as the ASE filter.
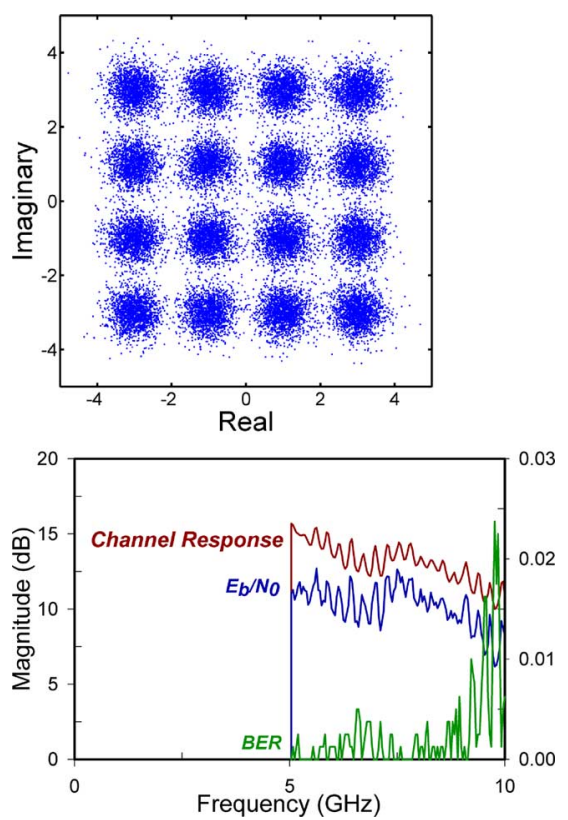

Fig. 5. Results for $20 \mathrm{Gbit} / \mathrm{s}$ digital system. Equalized constellation channel response, $\mathrm{E}_{\mathrm{b}} / \mathrm{N}_{0}$ and BER.

A 512-point, nonoversampled, IFFT was used which, allowing for Hermitian symmetry and a guard band, gives 128 independent data carrying subcarriers. The output signal requires a 5- to 10-GHz bandwidth which exceeds the 5.8-GHz bandwidth limit specified for the AWG. This experiment is only possible if the AWG DACs are set to produce return-to-zero pulses for each sample rather than holding their output values 


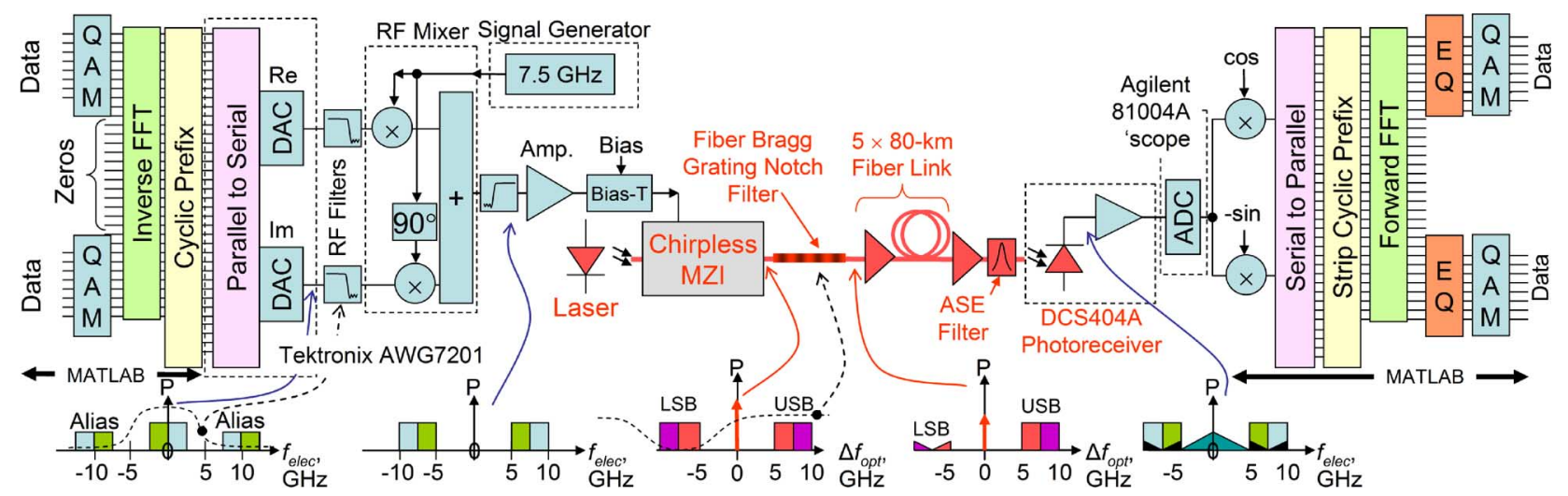

Fig. 6. $10 \mathrm{Gbit} / \mathrm{s}$ RF electrical upconverted system.
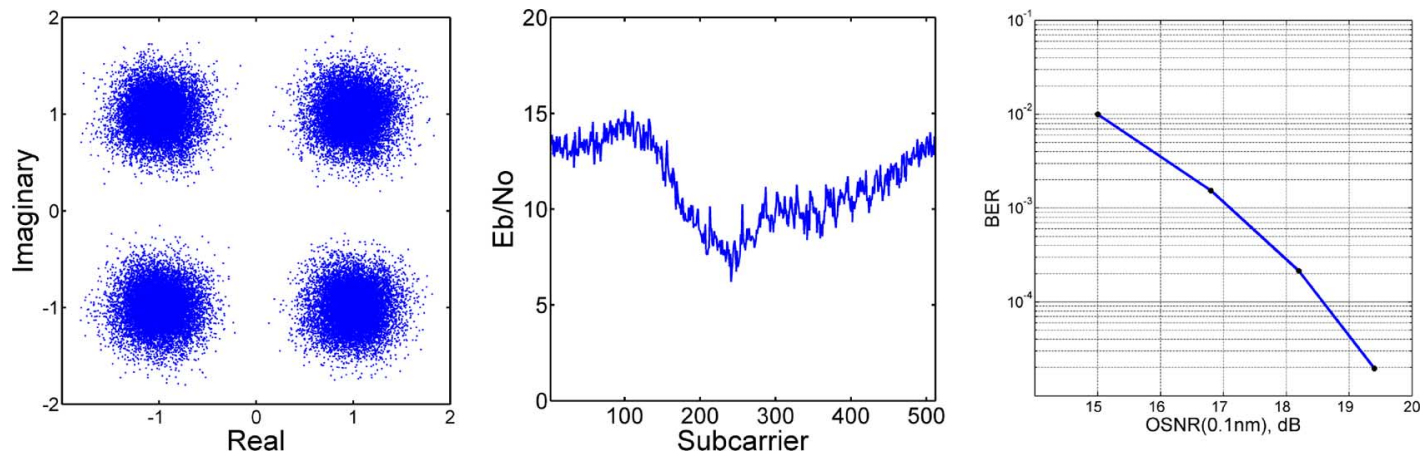

Fig. 7. Results for 10-Gbit/s system using RF upconversion. Equalized constellation. $E_{b} / N_{0}$ versus Subcarrier - High frequencies in middle subcarriers. BER versus OSNR.

for the duration of the sample. The specified bandwidth is still $5.8 \mathrm{GHz}$ but the rate of roll-off above this limit is reduced and a response at $10-\mathrm{GHz}$ of minus $10 \mathrm{~dB}$ is achieved. However, this mode introduces spurious noise and frequency response aberrations. A constellation size of 16QAM was used for a data rate of $20 \mathrm{Gbit} / \mathrm{s}$. Two hundred symbols (102 $000 \mathrm{bits}$ ) were transmitted over a distance of $320 \mathrm{~km}$ with a BER of $2.5 \times 10^{-3}$ at an OSNR of $26 \mathrm{~dB}$.

Fig. 5 shows the equalized constellation, the signal to noise ratio $\left(E_{\mathrm{b}} / N_{0}\right)$, the relative channel response and the BER versus frequency. The frequency response ripples are due to the AWG in return-to-zero mode and the bit errors can be seen to line up with the frequency response dips. A direct, back to back, electrical connection between the AWG and the DSO resulted in a BER of $3 \times 10^{-4}$ and an OSNR of $26 \mathrm{~dB}$ was required for a system BER to $2.5 \times 10^{-3}$.

\section{10-Gbit/s Up-Converted System}

Fig. 6 shows the experimental set up for the RF electrical upconverted system which uses the second transmitter design. The OFDM signal is modulated onto a 7.5-GHz electrical carrier before driving the optical modulator. The main limitation in this system is the bandwidth of the RF I/Q mixer.

A 512 subcarrier, $2 \times$ oversampled, trigonometrically interpolated [24] OFDM signal is generated by using a 1024 point IFFT with zero padding at the center. In contrast with the previous digital systems, all 512 subcarriers are modulated. The two output signals from the AWG each have a useful bandwidth of $2.5 \mathrm{GHz}$. These are low pass filtered to remove images and modulated onto a 7.5-GHz RF-carrier using an I/Q mixer. The bandwidth after up conversion is 5 to $10 \mathrm{GHz}$. The signal is amplified to drive a single input modulator and an FBG in transmission mode is used to suppress one sideband. Because of the imperfections of the RF electronics, only 4-QAM modulation could be supported and so the data rate was limited to $10 \mathrm{Gbit} / \mathrm{s}$. Five spans of $80 \mathrm{~km}$ of Corning SMF-28e fiber are used.

Fig. 7 shows the equalized constellation, $E_{\mathrm{b}} / N_{0}$ as a function of subcarrier and the BER versus OSNR. The dip in high frequencies (the center of the channel response graph) increased the required OSNR for a given BER. Nevertheless, because 4-QAM modulation was used, the required OSNR for a BER of $1 \times 10^{-3}$ was $17.2 \mathrm{~dB} ; 5 \mathrm{~dB}$ lower than the 12 -Gbit/s digital system which used 32 QAM.

\section{Channel Stability}

One of the advantages of DD-OOFDM compared with CO-OFDM is the relative stability of the channel and the reduced overheads in terms of training symbols and pilots. In this section we show that the channels in the experiments were very stable and that as a result, only infrequent training symbols are required.

The channel stability of the 12 -Gbit/s digital system is displayed in Fig. 8, which shows the unequalized amplitude of all data carrying subcarriers for all 300 received symbols as a function of frequency and time. The frequency axis shows the amplitude response of the subcarriers in the 2.4 to $4.8 \mathrm{GHz}$ spectrum and the time axis shows the subcarriers amplitude response over a time period of $15.36 \mu$ s, equivalent to the transmission of 300 


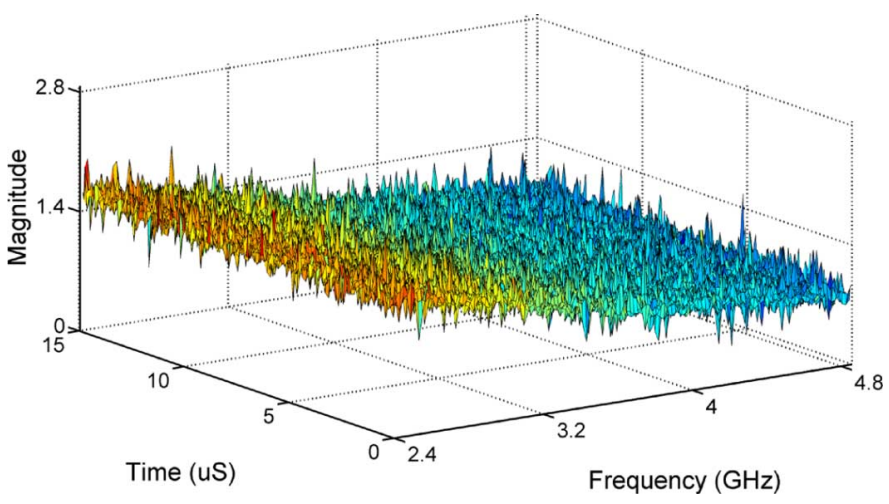

Fig. 8. Channel stability.

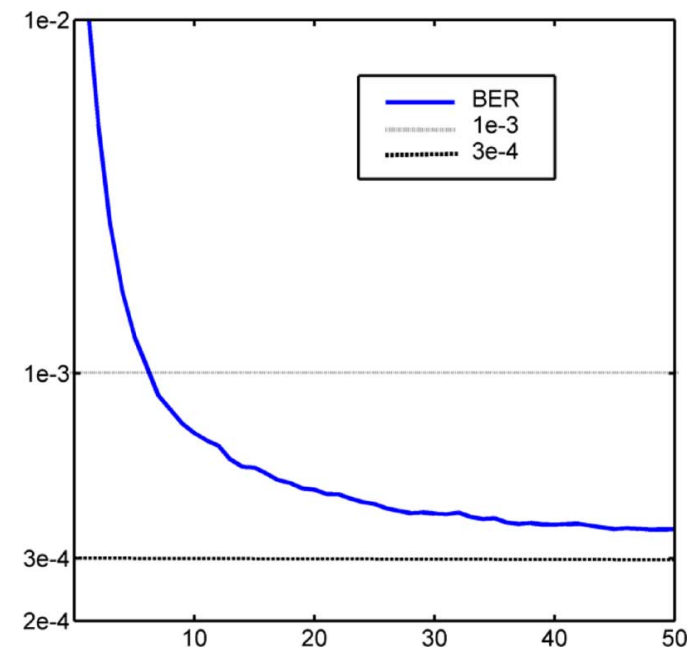

Fig. 9. Training overheads.

data symbols. While the falling amplitude response shown in Fig. 4 is apparent on the frequency response axis, the amplitude response for all subcarriers is constant with time except for the random fluctuations caused by the ASE noise.

In the experiments presented in Section III, the channel estimate was calculated by comparing the received OFDM symbols with the known transmitted OFDM symbols to find a series of channel responses. The channel estimate was than calculated by averaging over the responses to find the channel estimates for each subcarrier. The averaging reduces the effect of noise on the channel estimate and hence, the noise on the equalized data. The data was then reprocessed using this channel estimate.

The amount of averaging required for a low noise estimate is shown in Fig. 9 for the 12-Gbit/s system at a measured OSNR of $25 \mathrm{~dB}$. If seven channel responses were averaged to calculate the channel estimate, the equalized data had a BER of $1 \times 10^{-3}$. If 50 channel responses were averaged to calculate the channel estimate, the equalized data had a BER that reduced to $3.1 \times 10^{-4}$. Further averaging provided only negligible improvements.

To determine the training overheads required for the equalizer, the long term stability of the channel must be known, however the memory depth of the DSO limited the data capture lengths to 300 symbols. To remove this limitation, the same data sets were captured approximately three minutes apart and the channel estimate calculated in the first data set was used to

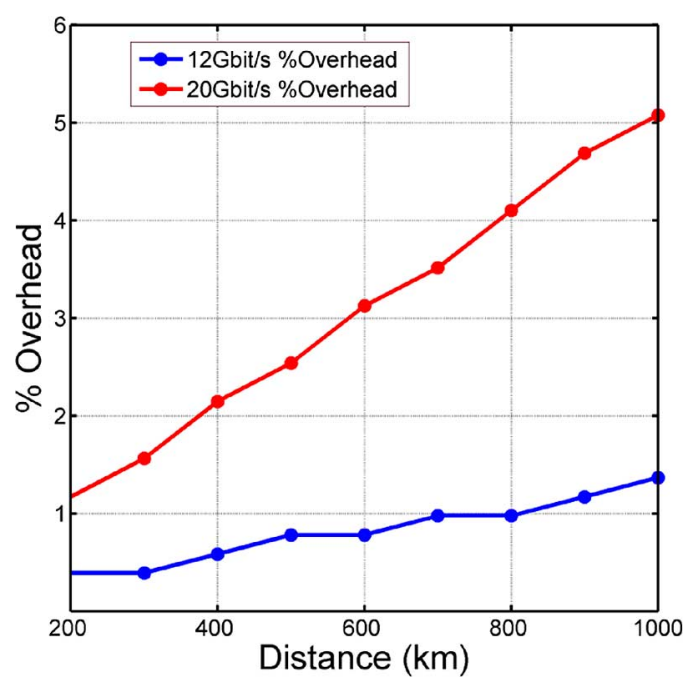

Fig. 10. Cyclic prefix for the 12- and 20 -Gbit/s systems.

equalize the data in the second data set. With the frame timing offsets aligned, the channel estimates from the first data set equalized the data in the second data set and the BER increased from $3.03 \times 10^{-4}$ to $5 \times 10^{-4}$ at an OSNR of $25 \mathrm{~dB}$. This process was repeated three times with similar results. The time between the data captures was approximately three minutes. This means that the frequency of training is in the order of once per one billion data symbols. Training overheads are negligible for the digital DD-OOFDM systems as tested in the laboratory conditions.

\section{CYCLIC PREFIX}

The length of the cyclic prefix (CP) needed to compensate for the effects of chromatic dispersion depends on both the length of the fiber and the bandwidth of the signal. Fig. 10 shows the length of $\mathrm{CP}$ required to compensate for chromatic dispersion in the 12- and 20-Gbit/s systems presented in this paper and for their specific configurations. The length of the $\mathrm{CP}$ is presented as a ratio of the required $\mathrm{CP}$ length (rounded up to the nearest integer) and the symbol length of 512 samples. Other dispersive sources such as transmitter and receiver filtering have been omitted from Fig. 10 for clarity but would need to be accounted for in a real system. For both experimental systems, a CP length of 32 samples equivalent to $3.2 \mathrm{~ns}$ for the $12 \mathrm{Gbit} / \mathrm{s}$ system and $1.6 \mathrm{~ns}$ for the $20 \mathrm{Gbit} / \mathrm{s}$ system was used for an overhead of $6.25 \%$. From Fig. 10, this CP is long enough to compensate the chromatic dispersion for both systems for a fiber distance exceeding $1000 \mathrm{~km}$. The $\mathrm{CP}$ was also used to compensate for some reflections within the system.

For distances significantly longer than $1000 \mathrm{~km}$, the CP length for the higher bandwidth 20-Gbit/s system would need to be increased.

\section{OSNR REQUIREMENTS}

Simulations with VPItransmissionMaker were performed to investigate the OSNR requirements without the limitations of the components used in the experiments for both the digital and 
TABLE I

SiMULATED OSNR(0.1 NM) REQUIREMENTS FOR BER $=1 \times 10^{-3}$

\begin{tabular}{|c|c|c|c|c|}
\hline $\begin{array}{c}\text { Sample Rate } \\
\text { Digital } \\
\text { (GS/s) }\end{array}$ & $\begin{array}{c}\text { Sample Rate } \\
\text { Up-converted } \\
\text { (GS/s) }\end{array}$ & QAM & $\begin{array}{c}\text { OSNR } \\
\mathbf{( 0 . 1 n m )}\end{array}$ & $\begin{array}{c}\text { Bit Rate } \\
(\mathbf{G b i t} / \mathbf{s})\end{array}$ \\
\hline 10 & 2.5 & 4 & 5.10 & 5 \\
\hline 20 & 5.0 & 4 & 8.27 & 10 \\
\hline 40 & 10 & 4 & 11.26 & 20 \\
\hline 10 & 2.5 & 16 & 11.83 & 10 \\
\hline 20 & 5 & 16 & 14.69 & 20 \\
\hline 40 & 10 & 16 & 17.89 & 40 \\
\hline 10 & 2.5 & 32 & 14.7 & 12.5 \\
\hline 20 & 5 & 32 & 17.7 & 25 \\
\hline 40 & 10 & 32 & 20.9 & 50 \\
\hline
\end{tabular}

the up-converted systems. The results in Table I show the required OSNR for a BER of $1 \times 10^{-3}$ for various sampling rates and constellation sizes. A carrier to sideband power ratio of 1:1 was used. A narrow bandwidth ASE filter was aligned with the carrier to reject the noise from the suppressed sideband and the bandwidth was $20 \%$ wider than the carrier and single sideband bandwidth for all simulations.

The simulations showed that a significant sensitivity improvement can be realized when a tighter ASE filter is used. For example, a simulation using a $12-\mathrm{GHz}$ filter reduced the required OSNR by $4.8 \mathrm{~dB}$ compared to the 200-GHz ASE filter used in the 4-QAM experiment.

\section{CONCLUSION}

We have demonstrated that DD-OOFDM with OSSB transmission can compensate for chromatic dispersion in long haul optical fiber links. All of the experiments used a simple direct detection photo-receiver. Three different transmitter designs were used. Two digital designs performed all signal processing functions in the digital domain. The bit rate of the digital systems were limited by the DAC speeds to $12 \mathrm{Gbit} / \mathrm{s}$ with 32-QAM modulation for the colorless transmitter and $20 \mathrm{Gbit} / \mathrm{s}$ with 16-QAM for the system which used an optical filter. The third transmitter design used an analog mixer to up-convert the signal so that the DAC requirements were reduced and $10 \mathrm{Gbit} / \mathrm{s}$ using 4-QAM was achieved.

The system overheads were very low. The use of direct detection resulted in a very stable channel for the two digital systems and as a consequence, a channel estimate from a single training sequence could be used to equalize billions of data symbols. This is in contrast to other forms of optical OFDM where significant overheads are required for pilot tones and sophisticated tracking algorithms are required to follow laser variations.

All of the systems were constructed using standard off-theshelf commercial components. Simulation results are presented to show the required OSNR for ideal components and the possible trade-offs between OSNR, DAC speed and constellation size.

\section{REFERENCES}

[1] H. Bulow, "Electronic dispersion compensation," in Opt. Fiber Commun. Conf., Anaheim, CA, 2007, OMG5.
[2] B. J. C. Schmidt, A. J. Lowery, and J. Armstrong, "Experimental demonstrations of $20 \mathrm{Gbit} / \mathrm{s}$ direct-detection optical OFDM and 12 Gbit/s with a colorless transmitter," in Opt. Fiber Commun. Conf., Anaheim, CA, 2007, PDP18.

[3] A. J. Lowery, L. B. Y. Du, and J. Armstrong, "Performance of optical OFDM in ultralong-haul WDM lightwave systems," J. Lightw. Technol., vol. 25, no. 1, pp. 131-8, Jan. 2007.

[4] S. L. Jansen, I. Morita, N. Takeda, and H. Tanaka, "20-Gb/s OFDM transmission over 4,160-km SSMF enabled by RF-pilot tone phase noise compensation," in Opt. Fiber Commun. Conf., Anaheim, CA, 2007, PDP15.

[5] B. Djordjevic, "PMD compensation in fiber-optic communication systems with direct detection using LDPC-coded OFDM," Opt. Express, vol. 15, no. 7, pp. 3692-3701, Apr. 2007.

[6] S. C. J. Lee, F. Breyer, S. Randel, M. Schuster, J. Zeng, F. Huiskens, H. P. A. van den Boom, A. M. J. Koonen, and N. Hanik, "24-Gb/s transmission over $730 \mathrm{~m}$ of multimode fiber by direct modulation of 850-nm VCSEL using discrete multi-tone modulation," in Opt. Fiber Commun. Conf., Anaheim, CA, 2007, PDP6.

[7] R. I. Killey, P. M. Watts, V. Mikhailov, M. Glick, and P. Bayvel, "Electronic dispersion compensation by signal predistortion using digital processing and a dual-drive Mach-Zehnder modulator," IEEE Photon. Technol. Lett., vol. 17, pp. 714-16, Mar. 2005.

[8] J. McNicol, M. O'Sullivan, K. Roberts, A. Comeau, D. McGhan, and L. Strawczynski, "Electrical domain compensation of optical dispersion [optical fibre communication applications]," in Opt. Fiber Commun. Conf., Anaheim, CA, 2005, OThJ3.

[9] T. H. Williams, "System for transmission of digital data using orthogonal frequency division multiplexing," U.S. Patent 5371 548, Dec. 6 , 1994.

[10] H. Hayashino, Y. Harada, T. Tomohiro, Y. Uno, and H. Oue, "Method of transmitting orthogonal frequency division multiplex signal, and transmitter and receiver employed therefore," U.S. Patent 5682 376, Oct. 28, 1997.

[11] P. M. P. Spruyt and P. P. F. Reusens, "Transmitter with phase rotor, modulator/demodulator, communication system and method performed thereby," U.S. Patent 6088 386, Jul. 11, 2000.

[12] R. Feced, R. Rickard, and E. Richard, "Reference phase and amplitude estimation for coherent optical receiver," U.S. Patent Applicat. 20050180760, Aug. 18, 2005.

[13] H. Kee, R. Epworth, A. Robinson, and R. Rickard, "Spectral shaping for optical OFDM transmission," U.S. Patent Applicat. 20050271387, Dec. 8, 2005.

[14] J. Armstrong, "Analysis of new and existing methods of reducing intercarrier interference due to carrier frequency offset in OFDM," IEEE Trans. Commun., vol. 47, pp. 365-9, Mar. 1999.

[15] Y. Xingwen, W. Shieh, and T. Yan, "Phase estimation for coherent optical OFDM,' IEEE Photon. Technol. Lett., vol. 19, pp. 919-21, Jun. 2007.

[16] J. Armstrong and A. J. Lowery, "Power efficient optical OFDM," Electron. Lett., vol. 42, no. 6, pp. 370-372, Mar. 2006.

[17] J. Armstrong, B. J. C. Schmidt, D. Kalra, H. A. Suraweera, and A. J. Lowery, "Performance of asymmetrically clipped optical OFDM in AWGN for an intensity modulated direct detection system," in IEEE Globecom 2006, San Francisco, CA, Mar. 2006, SPC07-4.

[18] X. Li, R. Mardling, and J. Armstrong, "Channel capacity of IM/DD optical communication system and of ACO-OFDM," presented at the ICC 2007, Glasgow, Scotland, 2007, unpublished.

[19] A. J. Lowery and J. Armstrong, " $10 \mathrm{Gbit} / \mathrm{s}$ multimode fiber link using power-efficient orthogonal-frequency-division multiplexing," Opt. Express, vol. 13, no. 25, pp. 10003-10009, Dec. 2005.

[20] M. R. D. Rodrigues and J. J. O'Reilly, "Assessment of the performance of radio over fiber based wireless networks employing OFDM signaling," in Proc. London Commun. Symp. 1999, London, U.K., Jul. 1999 , pp. $47-50$.

[21] P. Horvath and I. Frigyes, "Effects of the nonlinearity of a Mach-Zehnder modulator on OFDM radio-over-fiber transmission," IEEE Commun. Lett., vol. 9, pp. 921-923, Oct. 2005.

[22] A. J. Lowery and J. Armstrong, "Orthogonal-frequency-division multiplexing for dispersion compensation of long-haul optical systems," Opt. Express, vol. 14, no. 6, pp. 2079-2084, Mar. 2006.

[23] A. J. Lowery, L. Du, and J. Armstrong, "Orthogonal frequency division multiplexing for adaptive dispersion compensation in long haul WDM systems," in Opt. Fiber Commun. Conf., Anaheim, CA, 2007, PDP39.

[24] D. Fraser, "Interpolation by the FFT revisited-an experimental investigation," IEEE Trans. Acoust., Speech Signal Process., vol. 37, no. 5, pp. 665-75, May 1989. 


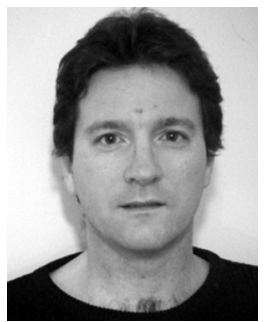

Brendon J. C. Schmidt received the B.Eng. degree (first class honors) in electrical and computer systems engineering from Monash University, Melbourne, Australia, in 2006.

$\mathrm{He}$ is currently working toward the M.Eng.Sci. degree at the same university. His research interests include OFDM in wireless and optical applications.

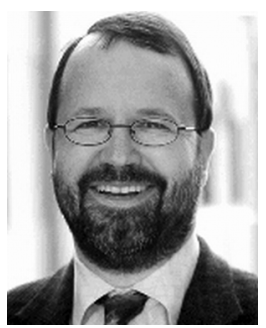

Arthur James Lowery (M'92-SM'96) was born in Yorkshire, U.K., in 1961. He received the B.Sc. degree (with first class honor) in applied physics from the University of Durham, Durham, U.K., in 1983 and the Ph.D. degree in electrical and electronic engineering from the University of Nottingham, Nottingham, U.K., in 1988.

From 1983 to 1984, he was with Marconi Radar Systems Ltd., U.K. In 1984, he joined the University of Nottingham as a Lecturer and pioneered time-domain field modeling of semiconductor lasers as the Transmission-Line Laser Model. In 1990, he emigrated to Australia to become a Senior Lecturer in the newly formed Photonics Research Laboratory, University of Melbourne, Melbourne. After working on photonic-CAD, packet switching and laser ranging, he was promoted to Associate Professor and Reader in 1993. He continued to develop novel time-domain simulation techniques and to lead collaborative research as a Fellow of the Australian Photonics Cooperative Research Centre. In 1996, he cofounded Virtual Photonics Pty. Ltd. with P. Gurney. In 1995, Optoelectronic, Photonic and Advanced Laser Simulator (OPALS) was released by Virtual Photonics (Melbourne, Australia), followed by Gigabit Optical Link Designer (GOLD) in 1996. He was the Chief Technology Officer with
Virtual Photonics when it merged with BNeD (Berlin) in 1998, which led to the development of VPI's physical-level photonic design automation tools such as VPItransmissionMaker. In 2004, he was appointed as a Professor with the Department of Electrical and Computer Systems Engineering, Monash University, Melbourne, where he is currently working on active photonic circuits, photonic systems, and applications of photonic technology. He has published more than 180 papers and four book chapters on the simulation of photonic devices and circuits and photonic applications such as mode locking, packet switching, transmission systems, and high-speed circuits.

Dr. Lowery was awarded the Peter Doherty Prize for Innovation, along with J. Armstrong, for their technical work on optical OFDM in June 2006. He is a Fellow of the Institute of Engineering and Technology, U.K.

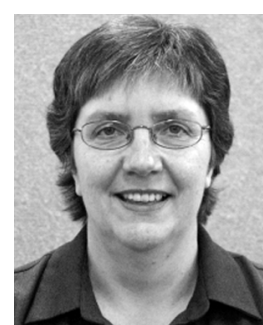

Jean Armstrong (M'89-SM'06) received the B.Sc. degree (first class honours) in electrical engineering from the University of Edinburgh, Scotland, in 1974, the M.Sc. degree in digital techniques from HeriotWatt University, Edinburgh, in 1980, and the Ph.D. degree in digital communications from Monash University, Melbourne, Australia, in 1993.

From 1974 to 1977, she worked as a Design Engineer with Hewlett-Packard Ltd., Scotland. In 1977. she was appointed Lecturer in Electrical Engineering with the University of Melbourne. Since 1977, she has held a range of academic positions with the University of Melbourne, Monash University, and La Trobe University. She is currently an Associate Professor with Monash University. Her research interests include digital communications, engineering education, and women in engineering. She has published over 100 papers including more than 70 on aspects of OFDM. Her work on OFDM has also led to a number of patents.

Dr. Armstrong was awarded the Peter Doherty Prize for Innovation, along with A. Lowery, for their work on optical OFDM in June 2006. She is Fellow of the Institution of Engineers Australia. 